\title{
Cooperative-hybrid detection of primary user emulators in cognitive radio networks
}

\author{
S. A. Adebo, E. N. Onwuka, A. U. Usman, and A. J. Onumanyi \\ Department of Telecommunication Engineering, Federal University of Technology, Nigeria
}

\begin{tabular}{l} 
Article Info \\
\hline Article history: \\
Received Mar 8, 2019 \\
Revised Nov 29, 2019 \\
Accepted Dec 10, 2019 \\
\hline
\end{tabular}

Keywords:

Cluster

Hybrid

Primary user emulator

Secondary users

Spectrum sensing

\begin{abstract}
Primary user emulator (PUE) attack occurs in Cognitive Radio Networks (CRNs) when a malicious secondary user (SU) poses as a primary user (PU) in order to deprive other legitimate SUs the right to free spectral access for opportunistic communication. In most cases, these legitimate SUs are unable to effectively detect PUEs because the quality of the signals received from a PUE may be severely attenuated by channel fading and/or shadowing. Consequently, in this paper, we have investigated the use of cooperative spectrum sensing (CSS) to improve PUE detection based on a hybrid localization scheme. We considered different pairs of secondary users (SUs) over different received signal strength (RSS) values to evaluate the energy efficiency, accuracy, and speed of the new cooperative scheme. Based on computer simulations, our findings suggest that a PUE can be effectively detected by a pair of SUs with a low Root Mean Square Error rate of 0.0047 even though these SUs may have close RSS values within the same cluster. Furthermore, our scheme performs better in terms of speed, accuracy and low energy consumption rates when compared with other PUE detection schemes. Thus, it is a viable proposition to better detect PUEs in CRNs.
\end{abstract}

Copyright $(2020$ Institute of Advanced Engineering and Science. All rights reserved.

\section{Corresponding Author:}

\author{
S. A. Adebo, \\ Department of Telecommunication Engineering, \\ Federal University of Technology, \\ Minna, Nigeria. \\ Email: adebosamuel@yahoo.com
}

\section{INTRODUCTION}

Cognitive Radio (CR) is an intelligent radio that automatically detects free channels (called white spaces or spectrum holes) and changes its transceiver parameters to transmit opportunisitically over these white spaces, while vacating occupied channels to prevent interference to existing primary users (PUs) [1-5]. A PU refers to the licensed owner of the spectrum (or channel) while we refer to a network of CR nodes as a CR network (CRN). CRNs provide several benefits to wireless communication such as improved quality of service by using free channels, longer transmission range over lower frequency bands, and improve spectra utilization [6]. Nevertheless, similar to other wireless communication networks, CRNs are also susceptible to security challenges [7].

A major security challenge in CRNs is the Primary User Emulator (PUE) attack. PUE refers to a situation in which a malicious secondary user (SU) or CR user feigns as a legitimate PU in order to deny other legitimate SUs in the CRN access to network resources, leading to denial of service or network flooding [8]. It is therefore pertinent for legitimate SUs to detect potential PUEs to prevent them from undermining the entire CRN. It is the quest to develop effective PUE detection methods that motivated the scheme proposed in this paper. To realize our quest, we considered the process of spectrum sensing (SS), which is pivotal to the success of CR technology. SS determines whether whitespaces exist or not in a sensed spectra $[9,10]$. SS is also an essential tool to determine whether a PUE exists or not in a CRN. It achieves this by allowing SUs to localize a potential PUE and to compare the PUE's location with the location of 
the legitimate PU. If discrepancies exist between the location of the PUE and the legit PU, then a PUE is considered to have been successfully detected by an SU and the base station can easily proceed to isolate it from the network [11]. However, since localization depends significantly on signals received from a potential PUE, it may become difficult to detect PUEs whose signals have been severely undermined by channel effects such as multipath fading and shadowing [12]. Consequently, cooperative spectrum sensing (CSS) has been adopted to mitigate such effects in CRNs. Here, SUs are configured to use CSS to make combined decisions concerning the presence of PUEs in a CRN $[13,14]$.

CSS in CRNs can be classified into distributed and centralized spectrum sensing [15-18]. In distributed spectrum sensing (SS), each SU performs spectrum sensing individually and communicates the sensed information to neighbouring SUs without common Fusion centre (FC). Distributed SS requires reliable communication links between the neighbouring SUs and incurs communication overhead during spectrum sensed data exchange. While in centralize SS, a Fusion centre (FC) gathers sensed information from all SUs in the CRN and uses these information to compute the sensing schedule of each SU over a particular channel, which makes it more efficient than distributed spectrum sensing [19-21]. CSS has been used for several purposes in CRNs, for example, authors in [16] proposed a centralized cooperating sensing scheme to estimate an optimal number of SUs and local sensing time that guarantees improve performance in terms of sensing delay and spectrum utilization. In [17], authors minimized interference to the PU while maximizing the expected transmission time in a CRN. They achieved this by determining the optimal decision threshold for a given false alarm probability using optimal combined rule in centralized cooperative sensing scheme. Similarly, authors in [22] proposed a reinforcement learning-based cooperative sensing (RLCS) to reduce detection overhead and improve detection performance in CRNs. According to [22], an FC cooperates with neighbouring SUs to determine an optimal set of cooperating SUs with minimum control traffic and less sensing delay. Essentially, we note that a significant amount of research has been done concerning the use of CSS in CRNs (see works in [23-26], however, most of these schemes focused mainly on maximizing sensing parameters in CSS. Others were concerned with optimizing the location of SUs to improve detection performance $[21,26]$.

However, there has been little or no work done concerning the use of CSS to effectively detect PUEs in CRNs. Consequently, in this paper, we have investigated a cluster-based centralized spectrum sensing scheme to detect PUEs with greater accuracy, speed and lower energy consumption rates. To achieve this, we clustered SUs into groups wherein SUs in the same cluster or neighbouring clusters typically experience similar signal propagation characteristics, which results to similar Received Signal Strength (RSS) for SUs in these cluster(s). In this case, we considered such similarly grouped SUs as closely related. We further introduced a hybrid scheme to better localize PUEs based on a combination of the angle of arrival (AoA) and received signal strength (RSS) methods. Our findings suggest that our scheme provides improved performance in detecting PUEs in CRNs. The rest of this paper is organized as follows. Section 2 presents the methodology and the model for investigating effects of cooperative sensing on the hybrid Localization Scheme for Detection of Primary User Emulator in CRNs. In section 3, we present the results and discussion. Performance analysis of our study and conclusion are presented in sections 4 and 5 respectively.

\section{METHODOLOGY}

In this section, we describe the system model in which our CSS based hybrid scheme is deployed. We present a general system model of the CRN, the CSS scheme based on energy detection, our hybrid scheme and the typical operations of the entire system are then presented in Figure 1.

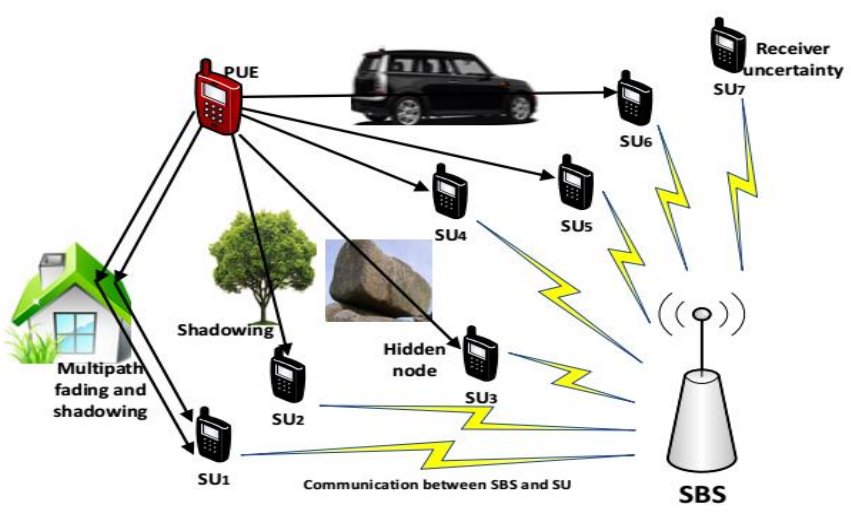

Figure 1. A typical CRN depicting SUs communicating with an SBS in the presence of a potential PUE 


\subsection{System model}

Figure 1 depicts a typical CRN consisting of several SUs set up to detect a PUE with several environmental obstacles existing in the environment that may cause fading and shadowing. To effectively detect the PUE, the SUs adopt CSS based on a hybrid scheme comprising of the received signal strength (RSS) and the angle of arrival (AoA) localization methods. Essentially, each SU receives different RSS values from the PUE. For example, the signal from the PUE to SU1 in Figure 1 is affected by the building and though no visible obstacle may exist between the PUE and SU4 or SU5, the RSS values may be different because of atmospheric conditions and the distance between nodes. We consider in Figure 1 a fusion centre (FC) depicted as the secondary base station (SBS) that coordinates the CSS scheme among the SUs. In this model, each SU senses the PUE's signal and reports its decision to the FC, which then conducts data fusion in order to make a final decision. This final decision is then broadcasted to the SUs after localization is concluded with the aim to isolate the PUE.

\subsection{Cooperative sensing scheme}

The CSS scheme consists of SUs that individually senses the PUE's signal energy and then each SU sends its local decision to the FC, which makes the final decision. We considered the energy detector (ED) as the spectrum sensing method since the PUE's signal energy is the only information available to each SU. Consequently, we modeled the signal energy of the PUE received at each ith SU as:

$$
x_{i}(m)= \begin{cases}u_{i}(m) & ; H_{0} \\ s_{i}(m)+u_{i}(m) & ; H_{1}\end{cases}
$$

where $\mathrm{m}=1,2, \ldots, \mathrm{N}$ is the time sample index and $\mathrm{N}$ is the total number of samples sensed by each SU, $\mathrm{x}$ is the signal received at the ith $\mathrm{SU}$, where $\mathrm{i}=1,2, \ldots, \mathrm{K}$, the PUE signal at each $\mathrm{SU}$ is denoted as s modeled as a variable with zero mean and variance $\sigma_{s}^{2}$, and ui(m) is modeled as Additive White Gaussian Noise (AWGN) with zero mean and variance $\sigma_{u}^{2}$. Here, K represents the number of SUs in the CRN, H0 and $\mathrm{H} 1$ represent the hypothesis that describes either the absence or presence of PUE signals in the CRN respectively.

Each SU receives xi(m) and computes a test statistic, which represents the signal energy as follows:

$$
T_{i}(X)=\frac{1}{N} \sum_{m=1}^{N}\left|x_{i}(m)\right|^{2}
$$

Thus, we computed the local probability of detection $p_{D}$ at each SU as:

$$
\begin{aligned}
& P_{D}^{i}=P\left(T_{i}(X)>\lambda_{i} / H_{1}\right) \\
& =Q\left(\frac{\left(\lambda_{i}-\left(\sigma_{s}^{2}+\sigma_{u}^{2}\right)\right) \sqrt{\frac{N}{2}}}{\left(\sigma_{s}^{2}+\sigma_{u}^{2}\right)}\right)
\end{aligned}
$$

and the probability of false alarm $P_{F A}$ at each SU as:

$$
\begin{aligned}
& P_{F A}^{i}=\operatorname{Pr}\left(T_{i}(X)>\lambda_{i} / H_{0}\right) \\
& =Q\left(\frac{\left(\lambda_{i}-\sigma_{u}^{2}\right) \sqrt{N / 2}}{\sigma_{u}^{2}}\right)
\end{aligned}
$$


The probability of missed detection $p_{M}$ can as well be calculated as:

$$
p_{M}^{i}=1-p_{D}^{i}
$$

where $Q(\bullet)$ is the Marcum Q-function given as:

$$
Q(x)=\frac{1}{\sqrt{2 \pi}} \int_{x}^{\infty} e^{-\frac{t^{2}}{2} d t}
$$

and $\lambda_{i}$ is the local detection threshold at each SU obtained from (4) as:

$$
\lambda_{i}=\frac{\sigma_{u}^{2} Q^{-1}\left(P_{F A}^{i}\right)}{\sqrt{\frac{N}{2}}+\sigma_{u}^{2}}
$$

Each SU then sends its local detection statistics to the FC, which plays a vital role in the CSS scheme. Based on the local statistics received at the FC from $K$ participating SUs, the FC denotes $\Lambda$ as the total number of SUs that have detected the PUE. It then adopts a decision strategy $\gamma$ described according to [27] as:

$$
\gamma= \begin{cases}H_{0}, & \text { if } \Lambda<M \\ H_{1}, & \text { if } \Lambda \geq M\end{cases}
$$

The FC decides on the final probability of detection and probability of false alarm based on $M$ different local statistics as follows:

$$
\begin{aligned}
& P_{D}=\sum_{m=1}^{K}\left(\begin{array}{l}
K \\
m
\end{array}\right) P_{D}^{m}\left(1-P_{D}\right)^{K-m} \\
& P_{F A}=\sum_{m=1}^{K}\left(\begin{array}{l}
K \\
m
\end{array}\right) P_{F A}^{m}\left(1-P_{F A}\right)^{K-m}
\end{aligned}
$$

\subsection{Hybrid localization scheme}

The FC uses the sensed information from each SU to localize the PUE. To achieve this, it adopts a hybrid of the RSS and angle of arrival (AoA) methods to detect the PUE. Figure 2 illustrates a setup of a number of SUs aiming to detect a PUE. Here, the FC groups the different SUs into respective pairs where each pair aims to detect the PUE. We describe the hybrid location scheme (HLS) for a particular pair as follows [28]: in Figure 3, let $\mathrm{x}_{1}, \mathrm{y}_{1}$ and $\mathrm{x}_{2}, \mathrm{y}_{2}$ denote the respective positions of $\mathrm{SU}_{1}$ and $\mathrm{SU}_{2}$. Similarly, let $r_{1}$ and $r_{2}$ represent the radii of the coverage areas of $S_{1}$ and $S_{2}$. Line D connects the centres of $S U_{1}$ and $\mathrm{SU}_{2}$, while $\varnothing$ and $\theta$ are the respective angles from which the legitimate PU's signal arrives at $\mathrm{SU}_{1}$ and $\mathrm{SU}_{2}$. The angles $\alpha_{1}$ and $\alpha_{2}$ represent the angles at which the PUE's signal arrives at $\mathrm{SU}_{1}$ and $\mathrm{SU}_{2}$. Let the position of the legitimate $\mathrm{PU}$ be $\left(\mathrm{X}_{\mathrm{PU}}, \mathrm{Y}_{\mathrm{PU}}\right)$ and the position of the PUE be $\left(\mathrm{X}_{\mathrm{e}}, \mathrm{Y}_{\mathrm{e}}\right)$. The Euclidean distance $\mathrm{D}$ between the pair of participating SUs is obtained as:

$$
\begin{aligned}
& D=\sqrt{\left(x_{2}-x_{1}\right)^{2}+\left(y_{2}-y_{1}\right)^{2}} \\
& Y_{e}-y_{1}=\left(X_{e}-x_{1}\right) \tan \alpha_{1} \\
& Y_{e}-y_{2}=\left(X_{e}-x_{2}\right) \tan \alpha_{2}
\end{aligned}
$$


$X_{e}=\frac{\left(x_{1} \tan \alpha_{1}\right)-\left(x_{2} \tan \alpha_{2}\right)+y_{2}-y_{1}}{\tan \alpha_{1}-\tan \alpha_{2}}$

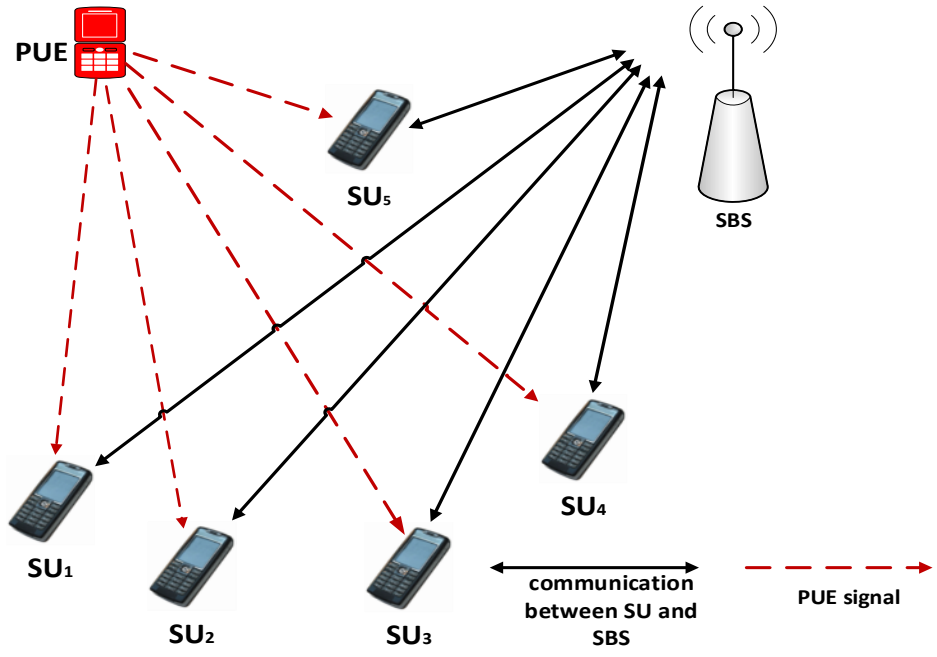

Figure 2. A setup of SUs cooperating to detect a potential PUE

Consequently, when a pair of SUs receive the signal from a potential PUE, first, each pair cooperates to compute the location of the transmitter using (14) and (15). Then, this estimated transmitter location is compared with the known location of the legitimate PU. If the transmitter's location is different from the legitimate PU's location, the transmitter is considered a PUE. Otherwise, it is considered as a legitimate PU and so the SUs quickly vacate the spectrum to avoid interference. The detection results are sent to the FC where final detection is concluded based on the decision strategy in (8). A simple strategy to cluster the SUs in a CRN is depicted in Figure 4. Here, the SBS forms four different clusters where each SU forms a pair and communicates this pairing information to the SBS. Essentially, an SU can form only one pair per time and in a situation where there are odd number of SUs in the CRN, the SBS simply excludes the last SU that fails to form a pair.

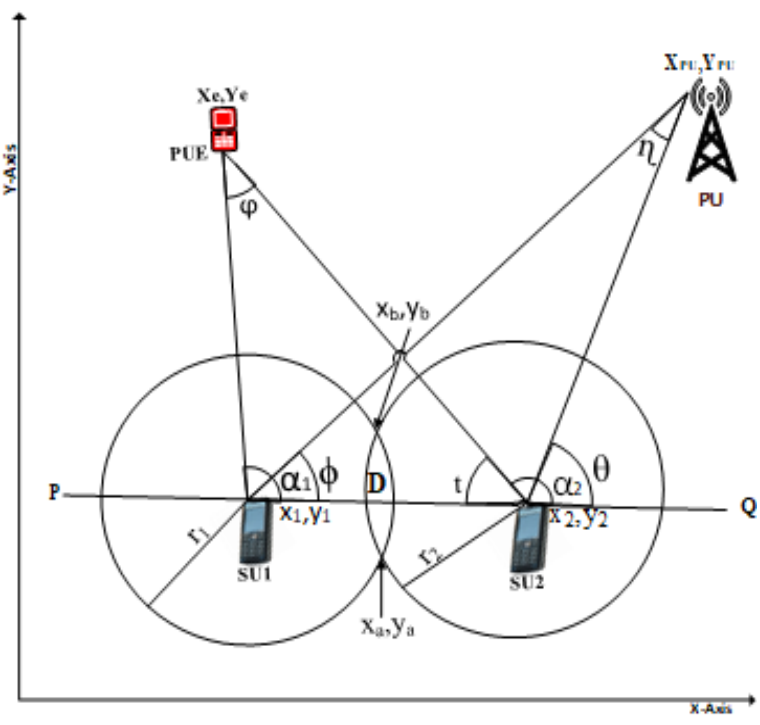

Figure 3. The two secondary users participating in the detection PUE

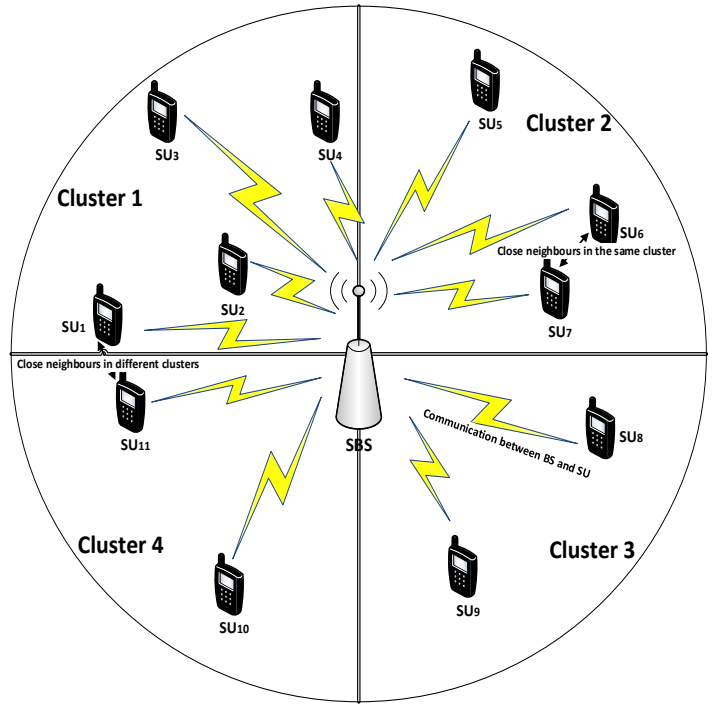

Figure 4. Clustering of SUs by a secondary base station (same as FC) 


\subsection{General operation of the cooperative-hybrid localization scheme (C-HLS)}

Essentially, each SU receives the RSS values from the PUE transmitter. Using the RSS, each SU estimates their respective distances from the PUE transmitter and the arrival angle of the signal using the HLS scheme. Our HLS is then used to localize the PUE based on the distance and the angular measurements. Different pairs of SUs distributed within clusters in the CRN are used to localize the PUE with the aim to increase detection accuracy. A pair of SUs is selected by the SBS using the RSS of each SU received within a power interval $[0, w]$ from K participating SUs. We describe a list of possible pairs that can be selected as follows:

- Two SUs with maximum RSS: In this case, the SBS selects from among all clusters the two nodes with the highest RSS values. This implies that the two nodes can be selected from different clusters.

- Two SUs with minimum RSS: The SBS selects two nodes with the lowest RSS values from among all clusters.

- Two SUs with medium RSS: The SBS computes the average RSS values from all nodes in the CRN and selects the two nodes having the closest values to the average RSS value.

- Two SUs with one having the highest RSS and the other having the lowest RSS: The SBS selects two nodes with one having the highest RSS and the other having the lowest RSS values in the CRN.

- Two SUs that are closely related: Here, the SBS selects two nodes with the two highest RSS values from the same cluster.

Our aim is to investigate the best pair of SUs that can most effectively detect the presence of PUEs in a CRN using our C-HLS scheme.

\subsection{Performance metric}

We evaluated the accuracy of the PUE localization scheme using the Root Mean Square Error (RMSE) function defined as:

$$
R M S E=\sqrt{\sum_{c=1}^{c} \frac{\left(L_{e s t}^{c}-L_{\text {real }}\right)^{2}}{c}}
$$

where $L_{e s t}$ and $L_{\text {act }}$ are the estimated and actual location of the PUE, and $C$ denotes the number of Monte Carlo trials over which the simulation was conducted.

\section{RESULTS AND ANALYSIS}

In this section, we discuss our findings concerning the use of the C-HLS over different pairselection schemes. Our simulation was conducted using MATLAB version 2017b. Here, SUs were randomly distributed over a spatial network of $100 \mathrm{~m}$ x 100m: The position of a pair of SUs relative to the PUE's location were varied over different Monte Carlo simulation averaged over 1000 trials (i.e. $\mathrm{C}=1000$ in $(15)$ ). The transmit power of the PUE was fixed at $50 \mathrm{dBm}$ and pathloss was computed using the free-space model for a reference distance of $1 \mathrm{~m}$ and loss exponent of 4, considering typical urban environments. Here, we note that schemes with lower RMSE values typically imply better accuracy.

Figure 5 presents the accuracy performance of the CSS-HLS using a pair of SUs with minimum and maximum, minimum, median, highest, and closely related RSSs. Our findings indicate that the accuracy of the C-HLS over different pair-selection schemes increases as the pair of SUs continuously recomputes the location of the PUE over time. As expected, Figure 5 shows that selecting two SUs with the highest RSS values converged to an RMSE value of 0.006 in 0.07 secs. Using this selection scheme implies that SUs that receive PUE signals via the best channels (least fading effects) generally leads to improved performance. Figure 5 further shows that the pair-selection scheme of SUs with the least (minimum) RSS values and the pair scheme with median RSS values typically converged to an RMSE value of 0.0081 and 0.0068 after 0.08 secs, respectively. This implies that using PUs with small RSS values (poor channel) conditions typically reduces PUE detection performance. The least performance occurred when using two SUs with maximum and minimum RSS values resulting in an RMSE value of 0.013 after 0.09 secs. This implies that using the minimum RSS values in a pair combination may not necessarily guarantee the best performance since detection performance may also be affected by poorly estimated AoAs, thus negatively affecting the performance of the pair scheme. An interesting finding in Figure 5 suggests that two SUs with closely related RSSs converged the fastest to an RMSE value of 0.0047 after 0.02 secs. This pair achieved the highest accuracy at the fastest rate because they had the highest RSS values from within the same cluster. Furthermore, since the two SUs with related RSS are not as far apart as the maximum RSS scheme from different clusters, they typically experience less pathloss leading to better performance than other schemes. 
Figure 6 presents the total energy consumed by each pairing scheme over the convergence time to their respective minimum RMSE values. Our findings indicate that the closely-related pairing scheme consumed the least energy because it converged fastest to its minimum RMSE value. Essentially, Figure 6 suggests two interesting observations as follows: the C-HSL scheme can be used based on the clustering approach as well as without clustering. In the clustering case, it is suggested that SU pairs with the highest RSS values should be selected from the same cluster, as this produces improved performance. However, in a non-clustered CRN, it is suggested that the maximum RSS pairing scheme should be adopted to achieve the best performance.

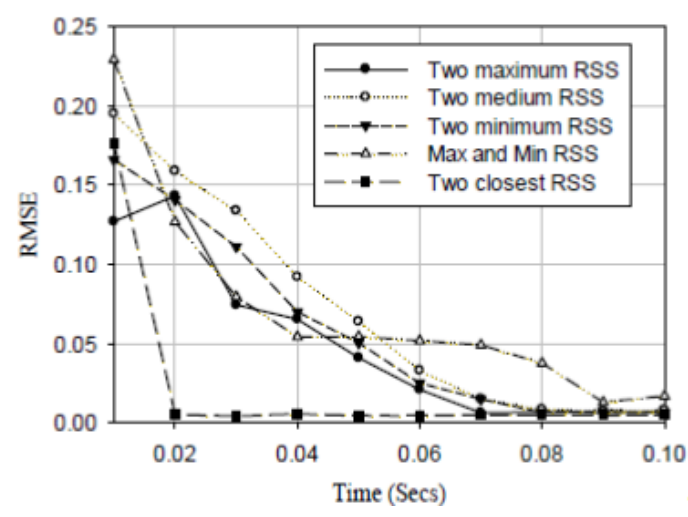

Figure 5. Comparative performance of the C-HLS scheme using two SUs with lowest, mediun, highest, closely related RSS

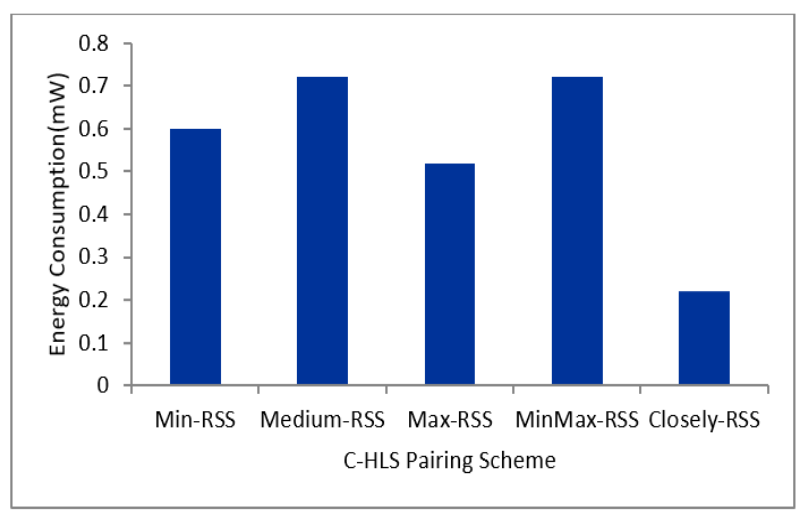

Figure 6. Energy Consumption of the C-HLS scheme using two SUs with lowest, median, highest, closely related RSSs

\subsection{Performance analysis}

The performance of the proposed Improved-hybrid Detection of Primary User Emulators in Cognitive Radio Networks was measured using root mean square error (RMSE) as shown in Figure 5. Similarly, performances of some schemes for the detection of primary user emulators in cognitive radio networks were evaluated using RMSE. The performance of the proposed Cooperative-hybrid Detection of Primary User Emulators in Cognitive Radio Networks is better than the performances of the hybrid scheme [28], AoA scheme [29], and RSS scheme [30] presented in Table 1. Notice that our cooperativehybrid scheme demonstrates higher accuracy than RSS, AoA hybrid of RSS and AoA as it exhibits the lowest RMSE of 0.0047. Moreover, it exhibits higher speed and energy efficiency than the methods used in [29, 30] as it takes lesser number of iterations to attain convergence. This results are quite significant because speed and accuracy are very important for efficient spectrum utilization. Furthermore, the need for energy efficiency cannot be overemphasized in realizing cognitive radio technology, considering the number of devices that will flood the network in future. Comparison of localization schemes shown in Table 1.

Table 1. Comparison of localization schemes

\begin{tabular}{ccc}
\hline Detection Scheme & Number of Iterations & RMSE \\
\hline RSS [29] & 50 & 0.2200 \\
AoA [30] & 30 & 0.0120 \\
The Hybrid of RSS and AoA [28] & 20 & 0.0050 \\
The Cooperative-hybrid Scheme & 20 & 0.0047 \\
\hline
\end{tabular}

\section{CONCLUSION}

In this paper, we have presented a cooperative-hybrid localization scheme (C-HLS) to improve PUE detection in CRN. The C-HSL scheme was investigated considering different pairing approaches with the aim to determine which pair achieves the best performance. We analyzed the C-HLS scheme based on the accuracy and energy consumption rate of the scheme as a function of time. Our findings indicate that two SUs with closely related RSS values best localizes a PUE in terms of accuracy, energy consumption and speed. Nevertheless, our scheme may benefit further from employing better spectrum sensing methods and incorporating adaptive threshold techniques in the $\mathrm{ED}$, which will be considered in future works. 


\section{ACKNOWLEDGEMENTS}

This research was supported by the TETFUND Institution-Based Research Intervention (IBRI) fund of the Federal University of Technology, Minna, Nigeria (TETFUND/FUTMINNA/2016-2017/6th BRP/15).

\section{REFERENCES}

[1] S. Srinu, S. L. Sabat, and S. K. Udgata, "Spectrum Sensing using Frequency Domain Entropy Estimation and its FPGA Implementation for Cognitive Radio,” Procedia Engineering, vol. 30, pp. 289-296, 2012.

[2] P. Lin, Y. Chen, P. Chang and S. Jeng, "Cooperative Spectrum Sensing and Optimization on Multi-Antenna Energy Detection in Rayleigh Fading Channel," 27th Wireless and Optical Communication Conference (WOCC), Hualien, pp. 1-5, 2018.

[3] A. J. Onumanyi, E. N. Onwuka, A. M. Aibinu, O. C. Ugweje, and M. J. E. Salami, “A Modified Otsu's Algorithm for Improving the Performance of the Energy Detector in Cognitive Radio," AEU-International Journal of Electronics and Communications, vol. 79, pp. 53-63, Sep. 2017.

[4] C. Xing, Z.-s. BIE, and W.-1. WU, "Detection Efficiency of Cooperative Spectrum Sensing in Cognitive Radio Network," The Journal of China Universities of Posts and Telecommunications, vol. 15, no. 3, pp. 1-7, 2008.

[5] A. Balieiro, P. Yoshioka, K. Dias, D. Cavalcanti, and C. Cordeiro, "A Multi-Objective Genetic Optimization for Spectrum Sensing in Cognitive Radio," Expert Systems with Applications, vol. 41, no. 8, pp. 3640-3650, 2014.

[6] A. J. Onumanyi, E. N. Onwuka, A. M. Aibinu, O. C. Ugweje, and M. J. E. Salami, "A Real Valued Neural Network Based Autoregressive Energy Detector for Cognitive Radio Application," International Scholarly Research Notices, vol. 2014, pp. 1-11, 2014.

[7] A. J. Onumanyi, A. M. Abu-Mahfouz, and G. P. Hancke, "A Comparative Analysis of Local and Global Adaptive Threshold Estimation Techniques for Energy Detection in Cognitive Radio,” Physical Communication, vol. 29, no. C, pp. 1-11, Apr. 2018.

[8] K. Akbari and J. Abouei, "Signal Classification for Detecting Primary user Emulation Attack in Centralized Cognitive Radio Networks," Electrical Engineering (ICEE), Iranian Conference on. IEEE, pp. 342-347, 2018.

[9] R. Yu, Y. Zhang, Y. Liu, S. Gjessing, and M. Guizani, "Securing Cognitive Radio Networks Against Primary user Emulation Attacks," IEEE Network, vol. 29, no. 4, pp. 68-74, 2015.

[10] Y.-C. Liang, Y. Zeng, E. C. Peh, and A. T. Hoang, "Sensing-Throughput Tradeoff for Cognitive Radio Networks," IEEE transactions on Wireless Communications, vol. 7, no. 4, pp. 1326-1337, 2008.

[11] E. Orumwense, O. Oyerinde, and S. Mneney, "Impact of Primary user Emulation Attacks on Cognitive Radio Networks," International Journal on Communications Antenna and Propagation, vol. 4, no. 1, pp. 19-26, 2014.

[12] A. Ashokan and L. Jacob, "Distributed cooperative spectrum sensing with multiple coalitions and non-ideal reporting channel," in Signal Processing, Informatics, Communication and Energy Systems (SPICES), IEEE International Conference on. IEEE, pp. 1-6, 2017.

[13] G. Sharma and R. Sharma, "Distributed Cooperative Spectrum Sensing Over Different Fading Channels in Cognitive Radio," in Computer, Communications and Electronics (Comptelix), 2017 International Conference on. IEEE, pp. 107-111, 2017.

[14] I. F. Akyildiz, B. F. Lo, and R. Balakrishnan, "Cooperative Spectrum Sensing in Cognitive Radio Networks: A Survey," Physical communication, vol. 4, no. 1, pp. 40-62, 2011.

[15] S. A. Alvi, M. S. Younis, M. Imran, et al., "A weighted linear combining scheme for cooperative spectrum sensing," Procedia Computer Science, vol. 32, pp. 149-157, 2014.

[16] D. Treeumnuk, S. L. Macdonald, and D. C. Popescu, "Optimizing performance of cooperative sensing for increased spectrum utilization in dynamic cognitive radio systems," in 2013 IEEE International Conference Communications (ICC) on IEEE, pp. 4656-4660, 2013.

[17] L. Yang, S. Song, and K. Letaief, "Optimizing Spectrum Sensing Efficiency in Cognitive Radio Networks," Computing, Communications and Applications Conference (ComComAp), IEEE, pp. 262-266, 2012.

[18] S. Shrivastava and D. Kothari, "Su throughput enhancement in a decision fusion based cooperative sensing system," AEU-International Journal of Electronics and Communications, vol. 87, pp. 95-100, 2018.

[19] G. Verma, V. Dhage, and S. S. Chauhan, "Analysis of Combined Data-Decision Fusion Scheme for Cognitive Radio," 2nd International Conference on Inventive Systems and Control (ICISC), IEEE, pp. 1324-1327, 2018.

[20] P. H. C. Souza, D. A. Guimar aes, and G. P. Aquino, "Efficient Fusion of Spectrum Sensing Information Under Parameter Uncertainty and Impulsive Noise," Journal of Communication and Information Systems, vol. 33, no. 1, 2018.

[21] M. Hajiabadi, H. Khoshbin, and G. A. Hodtani, "Cooperative Spectrum Estimation Over Large-Scale Cognitive Radio Networks," IET Signal Processing, vol. 11, no. 8, pp. 1006-1014, 2017.

[22] B. F. Lo and I. F. Akyildiz, "Reinforcement Learning for Cooperative Sensing Gain in Cognitive Radio Ad Hoc Networks," Wireless Networks, vol. 19, no. 6, pp. 1237-1250, 2013.

[23] H. Li, X. Cheng, K. Li, X. Xing and T. Jing, "Utility-Based Cooperative Spectrum Sensing Scheduling in Cognitive Radio Networks," Proceedings IEEE INFOCOM, Turin, pp. 165-169, 2013.

[24] A. Balieiro, P. Yoshioka, K. Dias, C. Cordeiro, and D. Cavalcanti, "Adaptive Spectrum Sensing for Cognitive Radio Based on Multi-Objective Genetic Optimisation," Electronics Letters, vol. 49, no. 17, pp. 1099-1101, 2013.

[25] P. Cheng, R. Deng, and J. Chen, "Energy-Efficient Cooperative Spectrum Sensing in Sensor-Aided Cognitive Radio Networks," IEEE Wireless Communications, vol. 19, no. 6, 2012. 
[26] F. Ye, X. Zhang, Y. Li, and C. Tang, "Faithworthy Collaborative Spectrum Sensing Based on Credibility and Evidence Theory for Cognitive Radio Networks," Symmetry, vol. 9, no. 3, pp. 36, 2017.

[27] W. Han, J. Li, Z. Tian, and Y. Zhang, "Efficient Cooperative Spectrum Sensing with Minimum Overhead in Cognitive Radio," IEEE Transactions on Wireless Communications, vol. 9, no. 10, pp. 3006-3011, 2010.

[28] S. A. Adebo, E. N. Onwuka, A. U. Usman and A. J. Onumanyi, "A Hybrid Localization Scheme for Detection of Primary User Emulator in Cognitive Radio Networks," International Journal of Computing and Digital Systems, vol. 8, no. 3, pp. 217-227, 2019.

[29] Y. A. Wassim Fassi Fihri, Hassan El Ghazi, Naima Kaabouch, Badr Abou El Majd "A Particle Swarm Optimization Based Algorithm for Primary User Emulation Attack Detection," IEEE 8th Annual Computing and Communication Workshop and Conference (CCWC), Las Vegas, USA, pp. 823-827, 2018.

[30] F. P. D. Cabric, "Cooperative DoA-Only Localization of Primary Users in Cognitive Radio Networks," EURASIP Journal on Wireless Communications and Networking, pp. 1-14, 2013.

\section{BIOGRAPHIES OF AUTHORS}
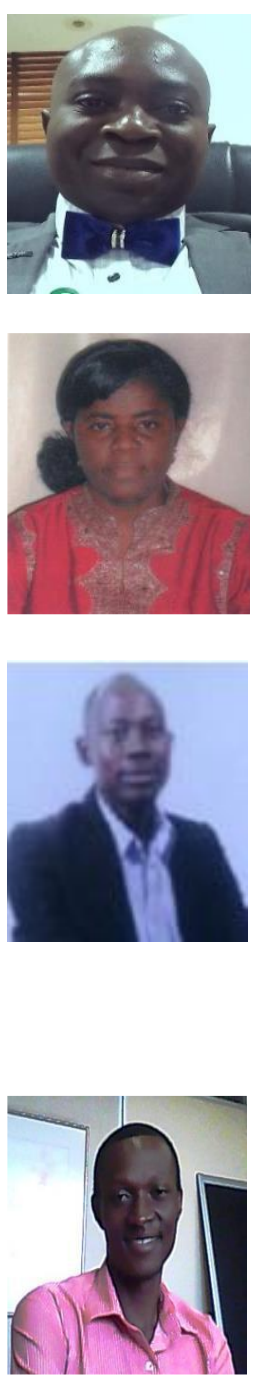

Samuel A. Adebo received B.Eng. degree in Electrical and Computer Engineering and M.Eng. degree in Communications Engineering from Federal University of Technology Minna, Nigeria in 2008 and 2013 respectively. He is currently working towards his Ph.D degree in Communications Engineering at the Department of Telecommunications Engineering, Federal University of Technology, Minna, Nigeria. His research interests include spectrum management in cognitive radio, and wireless sensor networks.

Elizabeth N. Onwuka is a Professor of Telecommunications Engineering. She holds a Ph.D. in Communications and Information Systems Engineering, from Tsinghua University, Beijing, People's Republic of China; a Master of Engineering degree, in Telecommunications; and a Bachelor of Engineering degree from Electrical and Computer Engineering Department, Federal University of Technology (FUT) Minna, Niger State, Nigeria. Her research interest includes Mobile communications network, Mobile IP networks, Handoff management, Paging, Network integration, Resource management in wireless networks, spectrum management, and Big Data Analytics.

A. U. Usman, is a Senior Lecturer with the Department of Telecommunication Engineering, Federal University of Technology, Minna, Nigeria. He obtained his B.Eng. degree in Electrical \& Computer Engineering from the same University in 1998. He acquired M.Sc. in Electrical Engineering from University of Lagos, Nigeria and $\mathrm{PhD}$ in Communication Engineering from Abubakar Tafawa Balewa University, Bauchi Nigeria in 2002 and 2014 respectively. $\mathrm{He}$ is currently the Deputy Dean, School of Electrical Engineering and Technology. $\mathrm{He}$ has teaching experience in the area of mobile radio propagation modeling, wireless communication system, wireless network resource utilization, numerical methods, and digital electronics. His research interest includes radio propagation modelling, indoor and outdoor wireless communication and application of Artificial Intelligent techniques in Engineering. He has published several papers in national/international journals and conferences.

Dr. Adeiza James Onumanyi received his B.Eng. degree in Electrical and Electronics Engineering from Abubakar Tafawa Balewa University, Bauchi, Nigeria, in 2005, and his M.Eng and $\mathrm{PhD}$ degrees in Communications Engineering from Federal University of Technology (F.U.T), Minna, Nigeria in 2010 and 2014, respectively. He has published several research articles in peerreviewed journals and in IEEE flagship conferences. Dr. Onumanyi lectures at the Department of Telecommunication Engineering, F.U.T, Minna, Nigeria. He has won grants at F.U.T, Minna, served on several conference-organizing committees. His research interests include spectrum sensing in cognitive radio, wireless sensor networks. 